\title{
Eventos no epilépticos psicógenos: El desafío constante de su diagnóstico y tratamiento.
}

\author{
Psychogenic non epileptic events: The constant challenge of its diagnosis and treatment.
}

\author{
Adderly R Quille Buendia ${ }^{1}$, Manuel Herrera Aramburu², Jorge G Burneo ${ }^{2}$
}

\section{RESUMEN}

Los eventos no epilépticos psicógenos (ENEP) son episodios paroxísticos descritos como cambios conductuales, alteraciones sensorio-sensitivas, o manifestaciones motoras que se asemejan a las crisis epilépticas pero sin mostrar actividad epileptiforme subyacente, no son producidos deliberadamente por el individuo, y responden a mecanismos multifactoriales de índole biopsicosocial. La información epidemiológica es limitada debido a la naturaleza heterogénea de la población afectada, así como a la escasa accesibilidad al videoelectroencefalograma (vEEG) necesario para su diagnóstico. En este contexto, es necesaria la convergencia de dos elementos importantes: historia clínica detallada (características clínicas sugerentes de ENEP e identificación de los factores asociados) y la ausencia de cambios electroencefalográficos en el vEEG durante el episodio. La evaluación psicológica provee información adicional relevante para la corroboración diagnóstica y para su manejo. El diagnóstico debe realizarse de manera oportuna, para evitar complicaciones en su evolución y tratamiento. Es vital una actitud positiva y alentadora hacia el/la paciente al momento de comunicarle su diagnóstico, así como la coordinación entre el neurólogo tratante y los profesionales de salud mental involucrados en el manejo y seguimiento del caso. La psicoterapia cognitiva conductual, asociada a psicofármacoterapia -cuando pertinente-, constituyen el tratamiento más eficaz de estos pacientes. Existen limitados estudios en Latinoamérica sobre aspectos epidemiológicos y clínico-evolutivos de pacientes con ENEP, por lo que más investigación y publicaciones respecto a esta problemática son indispensables dado su impacto sobre la calidad de vida de los pacientes y sobre el costo de su manejo en los sistemas de salud.

PALABRAS CLAVE: Crisis epiléptica, convulsiones no epilépticas, trastorno conversivo, psicoterapia, factores de riesgo, electroencefalografía.

\section{SUMMARY}

Psychogenic Non-Epileptic Events (PNEE) are paroxysmal episodes described as behavioral changes, sensorysensitive disturbances, or motor manifestations that resemble epileptic seizures without showing underlying epileptiform activity, are not deliberately produced by the individual, and respond to multifactorial biopsychosocial mechanisms. Epidemiological information is limited due to the heterogeneous nature of the affected population, and to the limited accessibility to the video electroencephalogram (vEEG) necessary for its diagnosis. In such context, the convergence of two important elements is necessary: a detailed clinical history (clinical characteristics suggestive of PNEE, and identification of the associated factors), and the absence of electroencephalographic "ictal" changes in the vEEG. A psychological evaluation provides additional information, relevant for the diagnostic corroboration and management. The diagnosis must be made in a timely manner, to avoid complications in its evolution and treatment. A positive and encouraging attitude to the patient is vital when informing him/her of the diagnosis, as it also is the coordination between the treating neurologist and mental health professionals involved in the management

\footnotetext{
1 Servicio de Neurología, Hospital Daniel Alcides Carrión, Universidad Nacional mayor de San Marcos. Lima, Perú

2 Programa de Epilepsia, Schulich School of Medicine and Dentistry, Western University. London, Canada.
} 
and monitoring of the case. Cognitive behavioral psychotherapy, associated with psychopharmacotherapy -when needed-- constitute the most effective treatment approach for these patients. There are limited studies on the current epidemiological and clinical evolution of patients with PNEE in Latin America, and additional research regarding this problem is much needed, given its impact on the quality of life of the patients and on the cost of its management in the health care systems.

KEYWORDS: Epileptic seizures, non-epileptic seizures, conversion disorder, psychotherapy, risk factors, electroencephalography.

\section{INTRODUCCIÓN}

Los eventos no epilépticos psicógenos son desórdenes neurológicos episódicos considerados como un subtipo de trastornos funcionales, caracterizados por paroxismos de alteración del comportamiento, de síntomas sensitivos o motores semejantes a crisis epilépticas (CE) pero sin la alteración neurobioquímica subyacente y por ende sin cambios electroencefalográficos de naturaleza epiléptica durante estos episodios $(1,2)$; estos eventos son la manifestación clínica de una probable desregulación de los circuitos neuronales de respuesta al estrés (3).

Los ENEP son el resultado de la interacción de mecanismos biosociopsicológicos frente a un estrés (interno y/o externo al individuo) significativo. El componente biológico viene determinado por el contenido genético y la expresión fenotípica del individuo; el componente social es determinado por las experiencias vividas, algunas veces traumáticas -especialmente en la niñez-; y el componente "psicológico" viene dado por la estructura psíquica del sistema afectivo que predispone una determinada respuesta emocional frente a los estímulos (4).

Los ENEP, a diferencia del desorden facticio o la simulación, no son "producidos conscientemente", por lo que "no es falso" o "simulado" por el paciente, además responden exitosamente a la psicoterapia (aisladamente o con psicofármacos) en la mayoría de casos (5).

El diagnóstico definitivo, rápido y oportuno es crucial, ya que muchos de estos pacientes podrían ser tratados erróneamente como aquéllos con epilepsia, sin considerar otros trastornos psicológicos o fisiológicos. Los retrasos en el diagnóstico también pueden conllevar a la perpetuación de estos eventos, a una mala calidad de vida, y al uso excesivo de los recursos de atención médica (6). Los ENEP representan un problema importante en el campo de la neurología y la psiquiatría, debido a los desafíos en su diagnóstico y tratamiento.

En este artículo, revisaremos las investigaciones recientemente publicadas en la población adulta, centrándonos en las características semiológicas que distinguen a los ENEP de las CE, los criterios diagnósticos, la coexistencia de los ENEP con otras comorbilidades neuropsiquiátricas, además de estudios neurobiológicos y psicopatológicos disponibles.

\section{Terminología}

Existe un amplio espectro de sinónimos en la literatura médica utilizados para referirse a estos eventos, que pueden ocasionar confusión entre médicos y pacientes (7). Tal vez esta diversidad de términos usados, podría deberse a una falta de definición y criterios diagnósticos aceptados internacionalmente. Por lo que la adopción de una terminología universal puede facilitar la comunicación entre epileptólogos, médicos con poca frecuencia de manejo de casos de epilepsia, personal de salud no médico y pacientes.

La literatura reciente ha abandonado el uso de terminologías antiguas, como "ataque o histeroepilepsia" o "pseudocrisis", debido a su ambigüedad y connotación peyorativa (8). La Liga Internacional contra la Epilepsia (ILAE) en el 2013, adoptó sistemáticamente el término de "crisis no epilépticas psicógenas" para referirse a este trastorno específico (9). Sin embargo, algunos autores consideran el uso alternativo de "eventos no epilépticos funcionales", haciendo referencia a una perturbación funcional, en oposición a una patología estructural. Además sugieren el uso del término de "eventos", para evitar el termino confuso de "crisis" ("seizure" en inglés), relacionado más a trastornos epilépticos (10). Por lo tanto, consideramos adecuado y válido el uso de "eventos no epilépticos psicógenos", diferenciándolo de los "eventos no epilépticos de origen fisiológico". 
Los eventos no epilépticos de origen fisiológico pueden incluir afecciones tanto neurológicas (parasomnias, ataque isquémico transitorio, temblor, mioclonías no epilépticas), como no neurológicas (anomalías metabólicas, ingestión tóxica y arritmias cardíacas) que podrían confundirse con crisis epilépticas o ENEP. En este contexto es importante considerar a los síncopes como eventos no epilépticos de origen funcional que pueden tener tanto causas neurológicas (síncopes reflejos) como causas no neurológicas (cardiogénicos) (11).

\section{Epidemiología}

Los datos epidemiológicos de pacientes con ENEP son escasos, limitados, desafiantes y, por definición, deben incluir solo a los que fueron evaluados completamente con videoelectroencefalograma (vEEG). Una publicación de la ILAE en el 2011, clasificó a estos eventos dentro los tres principales problemas neuropsiquiátricos luego del trastorno ansioso depresivo y el trastorno psicótico (12). Se ha informado que representan entre el 5 al $10 \%$ de los pacientes ambulatorios en centros de epilepsia y el $20-40 \%$ de los pacientes hospitalizados en unidades de monitoreo de epilepsia (13).

La incidencia de ENEP en países de ingreso económico alto, es de 4,9/ 100000 personas-año (1). La prevalencia es aún difícil de determinar, debido a la ausencia de estudios epidemiológicos formales y recursos insuficientes (retraso del diagnóstico, retiro de pacientes de los servicios médicos después del diagnóstico). Una estimación sistemática basada en el número de pacientes referidos a centros de epilepsia mostró un rango de prevalencia de 2-33/100 000 $(0,002-0,033 \%)$ (14). Hasta la fecha, no se han estudiado las diferencias comparativas en la incidencia y prevalencia de los ENEP en todo el mundo.

Los ENEP tienden a comenzar en edades tempranas, con una edad media de $31( \pm 15)$ años, aunque pueden presentarse a cualquier edad (15). Existe un predominio del género femenino (70-80\% mujeres). Las diferencias neurobiológicas, sociales y de vulnerabilidad pueden explicar el predominio en el género femenino (16). El retraso en el diagnóstico se estima que es de 7 a 8 años aproximadamente, aunque según un estudio reciente es de $0,6 \pm 0,2$ años (1). La proporción de pacientes con ENEP que simultáneamente padecen de epilepsia es variable oscilando entre el 5 y el $50 \%$. Datos adicionales refieren que la mayoría de pacientes afectados con ENEP son desempleados y el $46 \%$ habían sido diagnosticado previamente con ansiedad o depresión (1). Diversos estudios y revisiones sistemáticas como la de Jones evidencian que la calidad de vida es menor en los pacientes con ENEP que en aquellos con epilepsia (17). El impacto en la calidad de vida está determinado por la múltiple comorbilidad asociada (depresión, ansiedad, disociación, estrés postraumático, etc.) y que se refleja en sentimientos de baja autoestima, miedo, depresión, culpa, conductas de evitación, que a su vez conllevan a restricciones sociales y la consiguiente pérdida de las relaciones familiares, amicales, laborales y las tensiones financieras derivadas de éstas) $(6,17,18)$.

En la actualidad se registran pocos estudios en América Latina, cuyos datos epidemiológicos son mayoritariamente similares al de la literatura mundial $(19,20,21,22)$. El limitado número de investigaciones podría deberse a las brechas en cuanto a recursos tecnológicos, capacitación del personal de salud y el tratamiento insuficiente en este desorden. No existen estudios epidemiológicos de ENEP en el Perú.

\section{Factores físicos y psicológicos: modelo biopsicosocial}

¿Por qué se producen?

De acuerdo a diversos estudios realizados, la población con ENEP es muy compleja y heterogénea. Esto debido a la frecuente asociación de diversas afecciones neurológicas (lesiones craneales, déficits neuropsicológicos, epilepsia comórbida, discapacidad intelectual, entre otras patologías) (23), así como a una alta prevalencia de trastornos psiquiátricos comórbidos (trastornos del estado de ánimo, trastornos de ansiedad, trastorno de estrés postraumático, trastornos somatomorfos, trastornos disociativos y trastornos de personalidad); estos últimos, muchas veces en relación a antecedentes de abuso físico o sexual (descritos en el $50 \%-77 \%$ de esta población) (24). Es claro que no existe un único mecanismo o factor contribuyente que sea necesario o suficiente para desarrollar este desorden. Gran parte de estas investigaciones apoyan un modelo multifactorial, un enfoque biopsicosocial que explique la causa de estos trastornos (25). Estos factores están clasificados en 4 subgrupos, los cuales pueden interaccionar dinámicamente y estar presentes en un determinado paciente:

- Factores de predisposición: Aquellos que aumentan la vulnerabilidad al desarrollo de ENEP en posteriores etapas de la vida (factor genético femenino, abuso sexual infantil, abuso físico y negligencia infantil, epilepsia). 
- Factores precipitantes: Aquellos que ocurren días o meses previos al inicio de estos eventos (abuso sexual, lesión o experiencia traumática "simbólica" en la edad adulta, muerte o separación de familiares o amigos, pérdida de empleo, partos, procedimientos quirúrgicos, desastres naturales, lesión cerebral traumática).

- Factores desencadenantes: Aquellos que ocurren durante los segundos o minutos previos al evento (crisis de pánico, fobias, anestesia general, crisis focales epilépticas).

- Factores de perpetuación: Aquellos que hacen propensa la recurrencia de los eventos y por ende hacen difícil la recuperación o agravan esta condición (la evitación, el aislamiento, el desempleo, la depresión, ansiedad, retraso en el diagnóstico, errores de tratamiento, aspectos culturales del paciente) (26).

\section{Mecanismos psicopatogénicos: modelo cognitivo integrador}

¿Cómo se producen?

Aunque la neurobiología de los ENEP es poco conocida, existen enfoques que buscan explicar los mecanismos patogénicos implicados. Estudios funcionales de neuroimágenes (resonancia magnética funcional) sugieren que estos eventos son el resultado de una disfunción de redes cerebrales específicas y de conectividad anormal $(27,28)$. Estos modelos sugieren una alteración en las redes cerebrales involucradas en el procesamiento de las emociones (ínsula, corteza cingulada anterior y media, corteza orbito frontal) y el control ejecutivo (giro frontal inferior y corteza parietal) con otras responsables de los procesos sensorio-motores (surco pre y post central, regiones premotoras) y cognitivos (conexiones entre éstas y otras áreas) (28-30).

Es oportuno mencionar un estudio (3) que compara dos grupos: uno conformado por pacientes con diagnóstico definido de ENEP; y otro, por controles sanos (12 individuos en cada grupo). La principal variable sujeta a comparación fue la activación de las zonas afectivas (amígdala, hipocampo, ínsula), zonas de control ejecutivo (circunvolución frontal inferior y media) y zonas motoras (giros pre central y post central) durante el sometimiento a estrés externo - prueba escrita con cálculos numéricos, estímulos sonoros intermitentes durante ésta, además de la emisión de comentarios como "hay ciertas demoras en su respuesta" o anticiparles que se subiría el nivel de la prueba "ya que los niveles previos eran sencillos"- y durante el reposo. El principal hallazgo fue una hiporreactividad en amígdalas (29), además del hipocampo izquierdo durante el momento de estrés en el grupo de pacientes con ENEP. Por lo contrario, durante la evaluación en reposo, se observó un aumento de la conectividad entre la amígdala derecha y los giros frontal inferior y medio del lado izquierdo al igual que con el giro precentral izquierdo. En conclusión, se planteó que estos resultados, sumados a los de estudio previos (3), sugieren un desorden funcional, descrito como una desregulación en las redes de interconexión entre las cortezas afectivas, motoras y de control ejecutivo $(29,30)$.

Integrando esta información, se podría conceptualizar que los ENEP son episodios breves facilitados por una disfunción de los sistemas afectivocognitivo-motor y de control ejecutivo. Un modelo explicativo que hace un consenso de las teorías existentes e intenta proporcionar un marco único general que explique los casos de ENEP a pesar de su heterogeneidad clínica y psicológica, es el Modelo Cognitivo Integrador (MCI). Este modelo propone que los signos y síntomas de estos pacientes resultan de la ejecución automática de una representación mental aprendida de crisis, proceso llamado "andamio de crisis", que acontece en un contexto de disfunción inhibitoria emocional, que resulta del estrés crónico, la excitación y factores que comprometen un procesamiento de alto nivel. Este modelo psicopatológico integra los rasgos de vulnerabilidad identificados en los ENEP, propone la existencia oscilante y simultánea de respuestas de hiperexcitación (señaladas por hipervigilancia, preocupación somática, reactividad emocional y falta de regulación emocional); y respuestas de hipo excitación (caracterizadas por disociación, tendencias de evitación, alexitimia - una incapacidad para identificar y / o describir estados emocionales). El andamio de crisis, consiste en una secuencia de percepciones y actividades motoras inicialmente formadas por experiencias reflejas inherentes (congelación, sobresalto), síntomas físicos (pre-síncope / disociación / hiperventilación / lesión craneal), pero también conocimiento personal modelado $(30,31)$.

\section{Manifestaciones clínicas}

\section{Manifestaciones objetivas}

Las manifestaciones pseudo-ictales visibles o fisiológicas en pacientes con ENEP son diversas y con 
frecuencia se asemejan a las de las crisis epilépticas $\mathrm{u}$ otros eventos fisiológicos, así mismo se debe entender que ninguna característica semiológica es patognomónica en este trastorno (tabla 1).

Revisiones sistemáticas mostraron que los indicadores más sugerentes de ENEP son los episodios de duración prolongada, los movimientos aleatorios o de curso fluctuante, movimientos asincrónicos, el empuje pélvico, movimientos del cuerpo o de la cabeza de lado a lado, cierre ocular, llanto durante el episodio, recuerdo del período de aparente falta de respuesta (el paciente con ENEP "asiste a su crisis") y ausencia de confusión postictal (32). Otros signos menos confiables son: sentimientos subjetivos, automatismos, hiperventilación, la mordedura de la punta de la lengua, la cefalea periictal, el pseudosueño preictal y la incontinencia urinaria (33). Las crisis con rigidez y temblor, o las crisis con atonía son infrecuentes. Debido a que las publicaciones actuales han revelado que todos estos elementos clínicos no se combinan al azar, estos eventos pueden ser clasificados en 5

Tabla 1. Características clínicas de Epilepsia versus ENEP

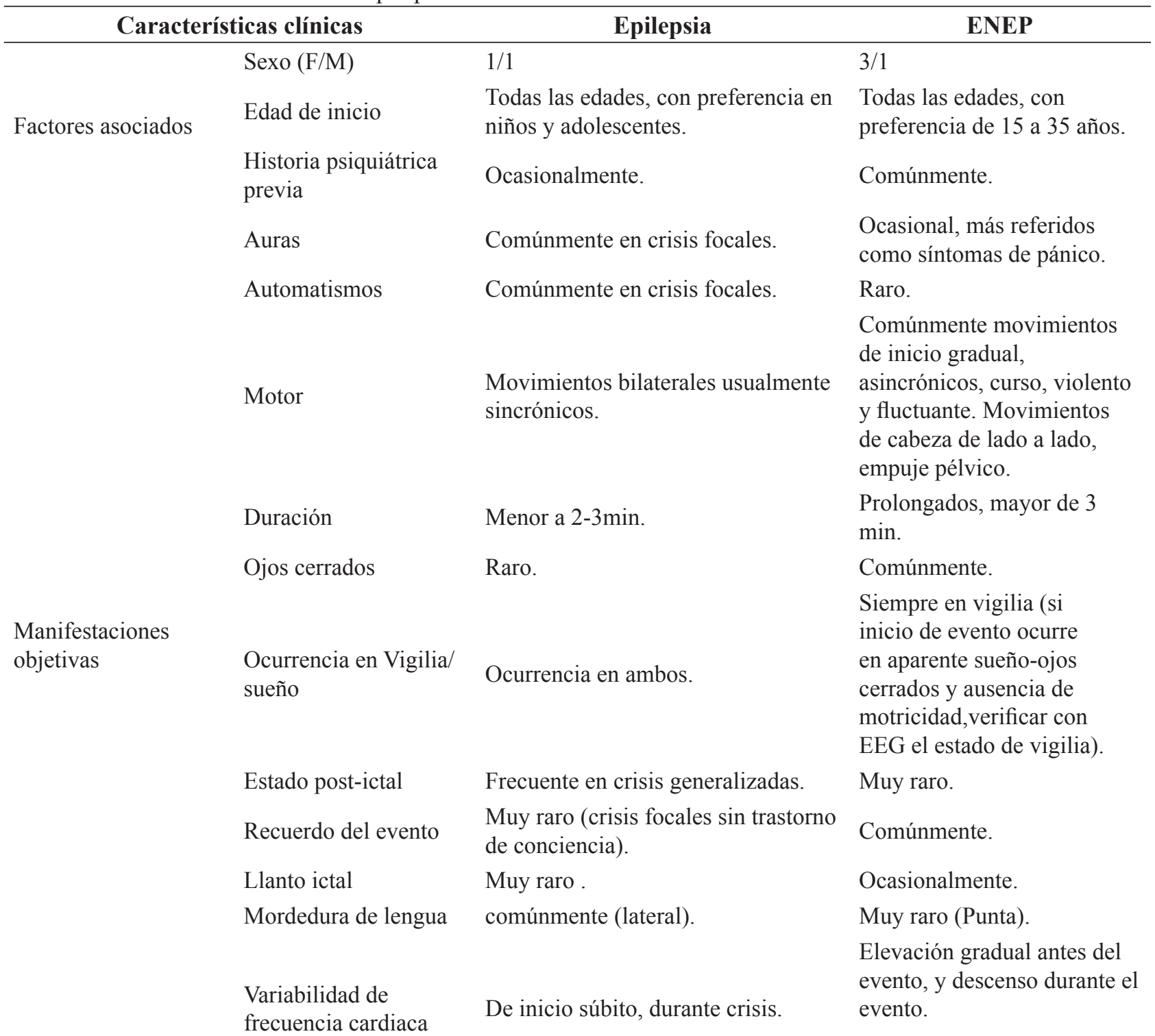

Manifestaciones

subjetivas Lenguaje narrativo Normalizan eventos.

Catastrofizan eventos.

Las cifras para la frecuencia: común $>30 \%$; ocasional $=10 \%-30 \%$; raro, $10 \%$; Muy raro, $<5 \%$. 
grupos, teniendo en consideración las características semiológicas principalmente visibles. Estas serían: crisis distónica con actividad gestual primitiva; crisis pauci-cinéticas con capacidad de respuesta conservada; pseudosíncope; crisis hipercinéticas prolongadas con hiperventilación y auras; y crisis prolongadas distónico-axiales (34). Si bien esta clasificación puede ser útil para un mejor entendimiento clínico, estas características pueden cambiar con el tiempo con mayor frecuencia que en los pacientes con crisis epilépticas. Sin embargo, las investigaciones recientes han demostrado que la semiología de los pacientes con ENEP es en realidad bastante estereotipada, al menos en un corto plazo, ya que estos eventos siguen un elemento condicionado, similar a un reflejo, que está adornado por el aprendizaje y la experiencia (35).

Dentro de las variaciones fisiológicas registradas en estos pacientes, resaltan aquellas derivadas de la activación del sistema nervioso autónomo caracterizadas por un tono parasimpático bajo con un tono simpático alto. La mejor reflejada es una variabilidad de la frecuencia cardiaca, que, a diferencia de las crisis epilépticas, tienen un aumento gradual justo antes del inicio de los eventos, y una disminución o alivio en el momento de la pseudocrisis (36).

\section{Manifestaciones subjetivas}

De la poca información disponible en este aspecto, se ha descrito que muchos de estos pacientes experimentan síntomas de pánico en forma de auras. Estas incluirían palpitaciones del corazón, sudoración, falta de aliento, sensación de asfixia, malestar en el pecho, mareos / inestabilidad, desrealización o despersonalización, miedo a morir, parestesias, escalofríos o sofocos que por lo general tienen una duración prolongada (varios minutos) a diferencia de las crisis epilépticas (usualmente segundos) (37).

Otro hallazgo importante es la manera como los pacientes con ENEP narran sus eventos, a menudo estos se enfocan preferentemente en las circunstancias en que ocurrieron, las consecuencias que estas producen o describen el evento como un espacio o lugar (por ejemplo, "entré en el ataque"), y a diferencia de los pacientes con epilepsia, estos hacen un esfuerzo considerable para explicar exactamente cómo se sienten en sus crisis ("la crisis me dejó inconsciente") (38). A menudo los pacientes que usan referencias de terceros para agravar de manera superlativa las experiencias de sus eventos tienen más probabilidades, en un $92.3 \%$, de tener ENEP (39).

\section{Diagnóstico y herramientas de diagnóstico}

Si bien el vEEG es crucial para el diagnóstico, no todos los pacientes tienen acceso inmediato a esta prueba. Por lo tanto, es clave realizar una historia clínica exhaustiva, que considere antecedentes, presencia factores de riesgo de epilepsia (crisis febriles complejas, antecedentes de traumatismo craneal significativo, antecedentes de infecciones cerebrales y antecedentes familiares de epilepsia), así como de factores biopsicosociales de ENEP (edad, sexo, historia de abuso físico o sexual, desordenes psiquiátricos, etc.). Énfasis en los desencadenantes y el patrón del evento (alta frecuencia de eventos con ingresos hospitalarios recurrentes a pesar del tratamiento), descripción de síntomas prodrómicos, semiología del período pre-inter y post- "crisis o evento" (un rápido retorno de la conciencia no es patognomónico de ENEP). Un relato del testigo del evento puede ser particularmente útil, aunque debe considerarse con cautela. Por lo tanto, cada característica clínica debe compararse y contrastarse (tabla 1)(40).

Considerando que la combinación del vEEG, la historia clínica y el relato de testigos ofrece un diagnóstico con altos niveles de certeza y excelente confiabilidad entre evaluadores (41). En el 2013, la ILAE publicó lineamientos sobre los requisitos mínimos para el diagnóstico de ENEP (tabla 2) (9). Este consenso sugiere cuatro categorías de certeza, basadas en diferentes combinaciones de datos de la historia del paciente, los informes de los testigos, las observaciones del médico clínico, así como el EEG ictal e interictal y el video ictal. Además, se debe tener en cuenta que un EEG interictal normal no excluye la epilepsia ni confirma ENEP. De manera similar, un EEG interictal anormal no confirma la epilepsia ni excluye a los ENEP. Otra advertencia es que los pacientes pueden tener epilepsia y ENEP concomitante. Para cumplir con los criterios de ENEP documentados, la historia clínica debe favorecer a ENEP y estos deben registrarse en el vEEG demostrando la ausencia de actividad epileptiforme ictal. Los niveles más bajos de certeza (clínicamente establecidos, posibles y probables) se basan en la disponibilidad de los componentes de diagnóstico.

\section{Monitoreo de vEEG ambulatorio/hospitalización}

Es la herramienta más importante a utilizar en la evaluación del paciente con sospecha de ENEP, es considerada la prueba "gold standard". El monitoreo de video-EEG puede ser realizada ambulatoriamente 
Eventos no epilépticos psicógenos: El desafío constante de su diagnóstico y tratamiento.

Tabla 2. Niveles diagnósticos de certeza para ENEP

\begin{tabular}{|c|c|c|c|}
\hline Nivel diagnóstico & Historia & Evento presenciado & EEG \\
\hline Posible & + & $\begin{array}{l}\text { Por testigo o descripción } \\
\text { autoinformado. }\end{array}$ & $\begin{array}{l}\text { No hay actividad epileptiforme en el EEG } \\
\text { interictal de rutina o privado de sueño. }\end{array}$ \\
\hline Probable & + & $\begin{array}{l}\text { Por el clínico que revisó la grabación } \\
\text { de video o en persona, mostrando la } \\
\text { semiología típica o PNES. }\end{array}$ & $\begin{array}{l}\text { No hay actividad epileptiforme en el EEG } \\
\text { interictal de rutina o privado de sueño. }\end{array}$ \\
\hline $\begin{array}{l}\text { Clínicamente } \\
\text { establecido }\end{array}$ & + & $\begin{array}{l}\text { Por un clínico con experiencia en el } \\
\text { diagnóstico de crisis epilépticas (en } \\
\text { video o en persona), que muestra una } \\
\text { semiología típica de PNES, mientras } \\
\text { que no esté en EEG. }\end{array}$ & $\begin{array}{l}\text { No hay actividad epileptiforme en el } \\
\text { EEG de rutina o ambulatorio durante } \\
\text { un evento típico, cuya semiología, en } \\
\text { caso de representar una crisis epiléptica, } \\
\text { necesariamente debería mostrar actividad } \\
\text { ictal en el EEG. }\end{array}$ \\
\hline Documentado & + & $\begin{array}{l}\text { Por un clínico con experiencia en } \\
\text { el diagnóstico de crisis epilépticas, } \\
\text { que muestra una semiología típica } \\
\text { de PNES, mientras que está en video } \\
\text { EEG. }\end{array}$ & $\begin{array}{l}\text { No hay actividad epileptiforme } \\
\text { inmediatamente antes, durante o después del } \\
\text { evento capturado en un video EEG con la } \\
\text { semiología típica de PNES. }\end{array}$ \\
\hline
\end{tabular}

Clave: +, características históricas consistentes con ENEP; EEG, electroencefalografía

LaFrance WC, Jr., Baker GA, Duncan R, Goldstein LH, Reuber M. Minimum requirements for the diagnosis of psychogenic nonepileptic seizures: a staged approach: a report from the International League Against Epilepsy Nonepileptic Seizures Task Force. Epilepsia. 2013;54(11):2005-18.

o en hospitalización, teniendo en cuenta que aquella realizada en una unidad de monitoreo ofrece pruebas clínicas (posibilidad de evaluar al paciente durante el evento por personal entrenado) y de seguridad adicionales(42). Algunas consideraciones que deben tenerse en cuenta en la lectura de los registros de vEEG es que deben ser examinados cuidadosamente por médicos capacitados, más aún si la estancia en una unidad de monitoreo es un recurso de difícil acceso y alto costo, además se debe capturar la mayoría de los tipos de eventos manifestados y siempre debe incluir el registro de electrocardiograma (ECG) continuo porque las arritmias cardíacas y la asistolia pueden conducir a una actividad convulsiva que puede confundirse con la epilepsia. Debe tenerse especial consideración en pacientes con crisis epilépticas focales frontales en donde la alteración del registro de EEG puede ser sutil y por ende constituir un reto diagnóstico (11). Similar precaución ha de tenerse en el registro de vEEG de superficie cuando el foco epileptogénico es profundo o superficial pero pequeño (menos de $6 \mathrm{~cm}^{2}$ ) (43). El vEEG ambulatorio es particularmente útil para aquellos pacientes con alta frecuencia de eventos estereotipados que sean pasibles de registro en un periodo corto de monitoreo. Sin embargo, es sabido que esa condición es poco frecuente y que el médico tratante muy posiblemente no tomará decisiones terapéuticas significativas con registros ambulatorios en pacientes con eventos de diferente semiología. Hasta la fecha, no se han identificado características EEG positivas que sean diagnósticas de ENEP. Aunque, un estudio piloto reciente mostró que la desincronización del ritmo $\beta$ en el EEG en pacientes con ENEP, podría ser un marcador de un próximo evento (44).

\section{Evaluación psicológica}

Una evaluación psicológica es primordial como parte de una estrategia de evaluación integral (45). Estas deben realizarse de manera complementaria a la evaluación clínica y el monitoreo con vEEG, ya que pueden ayudar en el diagnóstico, así como a dirigir el tratamiento futuro de estos pacientes. Algunos estudios revelan que la combinación de sub escalas del Inventario de evaluación de la personalidad (PAI) y del Inventario multifásico de personalidad 2 (MMPI-2), pueden ayudar a la distinción de ENEP y CE (sensibilidad del $86.7 \%$ y una especificidad del $82,4 \%$ ) (46), pero se recomienda que estas no se deben de utilizar de manera única para diferenciarlos.

\section{Neuroimágenes}

Actualmente los estudios de neuroimágenes, como la tomografía y resonancia magnética cerebral, son 
utilizados en la evaluación inicial de manera estándar ante la sospecha de crisis epilépticas. Por lo que las anomalías observadas en las imágenes deben interpretarse con precaución ya que no necesariamente diferencian entre eventos no epilépticos de crisis epilépticas. Si bien los estudios de neuroimágenes funcionales, pueden sugerir alteraciones en la conectividad cerebral de las regiones asociadas con la actividad motora, el procesamiento emocional y las funciones ejecutivas; no son de uso rutinario debido a evidencia insuficiente (47).

\section{Otras herramientas}

Los estudios de laboratorio podrían ser útiles, pero generalmente se realizan para excluir causas metabólicas o tóxicas (48). Se han estudiado otros marcadores de laboratorio que distingan el diagnóstico de ENEP y CE como el recuento de leucocitos, el dosaje de prolactina en suero, lactato sérico, la creatina quinasa y la enolasa específica de neuronas se han evaluado en pacientes con ENEP; sin embargo, su limitado poder discriminativo los hace poco confiables $(48,49)$.

Existe evidencia clara sobre el uso de la electromiografía en pacientes con ENEP, demostrando ser eficiente en la distinción de crisis epilépticas convulsivas de las crisis psicógenas no epilépticas $(50,51)$. Además, se reportan algoritmos diagnósticos validados y usados en la distinción de estos eventos, con una precisión diagnóstica del $95 \%$ (52).

\section{TRATAMIENTO}

El primer paso para el tratamiento de este trastorno es un diagnóstico correcto y temprano; de lo contrario podría ocasionar complicaciones iatrogénicas, así como resultados desfavorables a largo plazo. Proporcionar un diagnóstico definitivo; identificar factores predisponentes, precipitantes y perpetuadores; evaluar las comorbilidades neurológicas-psiquiátricas y conflictos sociales/familiares son esenciales para comprender al paciente con ENEP. Actualmente no existe directrices o protocolos universales sobre el manejo o tratamiento de estos pacientes, esto podría deberse a que existen estudios globales de bajo nivel de evidencia, o tal vez a que estos eventos se presentan en una población muy heterogénea. Un punto importante a considerar, es la participación activa y continua del paciente, la familia y de un equipo multidisciplinario (neurólogo, psiquiatra y otros profesionales de salud mental) (53). La participación activa de los neurólogos debe continuar más allá de la evaluación diagnóstica, en particular entre los pacientes con trastornos psiquiátricos graves. En la actualidad de manera general el manejo de pacientes con ENEP podría resumirse en cuatro fases (54) :

- Fase de comunicación del diagnóstico: la cual debe ser realizado por el neurólogo en compañía de la familia del paciente. Esta comunicación puede ser un desafío médico, debido a que la mayoría de los pacientes con ENEP suelen tener alexitimia asociada; reflejando menos probabilidades de aceptar, que sus experiencias negativas de vida, pueden tener una importancia gravitante en su enfermedad. Cada vez existe más evidencia que apoya que esta fase es un paso importante $y$ potencialmente eficaz en el tratamiento, con una consecuente reducción o detención de estos eventos. Por lo tanto, es crucial que el médico o el neurólogo explique al paciente bajo un enfoque honesto, positivo y alentador, logrando una "comprensión" adecuada sobre su desorden. Se debe utilizar terminología clara y métodos de comunicación según su experiencia clínica o estrategias desarrolladas según la literatura.

- Fase de compromiso:la cual es relevante, debido que un porcentaje considerable de pacientes con ENEP cuestionan el diagnóstico. En esta fase es importante el uso de habilidades clínicas (entrevistas motivacionales) que permiten adherir al apaciente al plan terapéutico y convencerlo de la necesidad del manejo simultáneo de los factores asociados (predisponentes, desencadenantes y perpetuadores) y de comorbilidades psiquiátricas, en caso lo hubiere, por un equipo multidisciplinario. El no lograr este paso puede llevar a una evolución clínica desfavorable y la búsqueda de nuevas consultas médicas, con la consiguiente exposición a ser sobremedicados, aumentar sus factores estresores y la cronificación del problema, además de aumentar los costos en los sistemas de atención de salud. Este paso puede ayudar a cambiar el curso y avanzar en su tratamiento. Al final de esta fase los pacientes aceptan el diagnóstico, evitan evaluaciones adicionales y comienzan a participar activamente en su tratamiento.

- Fase de intervención aguda: la cual comprende intervenciones a corto plazo como la psicoterapia y psicofarmacología. Esta fase tiene como objetivo principal la reducción de frecuencia de estos eventos. Pero, también se desea una mejora en las comorbilidades psiquiátricas, calidad de vida y recuperación funcional. La evidencia 
creciente muestra que terapia cognitiva conductual, las terapias psicodinámicas e interpersonales (por ejemplo, terapia familiar si existe una disfunción del sistema familiar) en asociación con psicofármacos (cuando es requerida) tienen una importante efectividad en el manejo de los ENEP. Con respecto a la farmacoterapia, incluye la reducción precoz e interrupción de los fármacos antiepilépticos (FAE) innecesarios (de manera gradual, dependiendo del fármaco) en pacientes con ENEP puros y una titulación mínima para disminuir los posibles efectos secundarios de estos FAE en pacientes con ENEP y epilepsia concomitante (mixto). De ahí la importancia del seguimiento por el neurólogo que hizo el diagnóstico. Finalmente, los medicamentos psicotrópicos (sertralina, venlafaxina) pueden ayudar a reducir los síntomas derivados de la ansiedad y la depresión, que comúnmente ocurren en los ENEP. Otro punto importante es involucrar a la familia del paciente la cual puede ayudar a su reintegración social.

- Lafase de intervención a largo plazo: la cual incluye la psicoterapia a largo plazo y manejo psicotrópico continuo de las comorbilidades psiquiátricas, entre otras. Si bien se conoce algunos indicadores de mal pronóstico, un subgrupo de pacientes continúa presentando estos eventos de forma crónica, buscando tratamiento en el departamento de emergencia y, lo que es más alarmante, una proporción continuará recibiendo atención inadecuada, por ejemplo, a través de la prescripción de FAE. A pesar de la disponibilidad de datos sobre los resultados a largo plazo en los ENEP, no existen estudios de tratamiento que se centren en el manejo a largo plazo de pacientes con síntomas prolongados.

\section{PRONÓSTICO}

El pronóstico de los pacientes con ENEP depende de diferentes factores, y el no identificarlos puede conllevar a un tratamiento refractario en más del $70 \%$ de estos pacientes. Dentro de los factores que pueden asociarse con un mejor pronóstico incluyen: educación superior, menor tiempo de diagnóstico desde el inicio de los síntomas, puntuaciones más bajas en las evaluaciones de somatización y escalas disociativas, ausencia de antecedentes de ansiedad o depresión, no dependencia de la seguridad social, ataques con características menos dramáticas, el género masculino (55). Otro estudio evidenció otros predictores adicionales de resultados deficientes los cuales incluyen: epilepsia concomitante o diagnósticos psiquiátricos, trastorno de personalidad, estado laboral, antecedentes de trauma psicológico relacionado a la salud, y visitas al servicio de urgencias al inicio del estudio (56).

\section{CONCLUSIONES}

Aunque en los últimos 25 años se ha logrado un significativo entendimiento sobre los ENEP, estos aún continúan siendo un desafío constante en el campo de la neurología y psiquiatría. Los ENEP se presentan en una población altamente heterogénea en cuanto a antecedentes, perfiles de personalidad, y comorbilidades asociadas. Diferentes modelos fisiopatogénicos han tratado de explicar el cómo y por qué ocurren estos eventos, pero la mayoría han concluido que se necesita la influencia de múltiples factores para explicar el origen de estos (modelo biopsicosocial, modelo cognitivo integrador). La neurobiología apoyada en estudios de neuroimágenes funcionales sugiere alteraciones específicas de redes neuronales implicadas en control de emociones, control ejecutivo, áreas motoras y cognitivas. Para lograr un diagnóstico eficaz se debe comenzar con la elaboración de una historia clínica detallada (enfocadas en la identificación de las características clínicas sugerentes de ENEP), sumada a la ausencia de actividad ictal durante los eventos registrados en el vEEG, además de una EP que sustente los factores de riesgo psicopatológicos. Este diagnóstico debe ser temprano, para evitar complicaciones en su recuperación. Una vez hecho el diagnóstico, la comunicación al paciente debe ser positiva y alentadora, logrando una coordinación entre los neurólogos y los expertos en salud mental para seguimiento.

Se debe incentivar la realización de mayor cantidad de estudios que reflejen la realidad latinoamericana, utilizando criterios diagnósticos universales (publicados por la ILAE), a pesar de la limitada accesibilidad al vEEG. El mayor conocimiento y mejor manejo de este desorden llevará a una gestión adecuada de recursos humanos y tecnológicos, disminuirá el impacto económico en los sistemas públicos de salud y mejora en la calidad de vida de nuestros pacientes.

\section{Correspondencia:}

Adderly Richard Quille Buendia.

Dirección: Calle los Rubíes 116, Urb. San Antonio, Bellavista-Callao, Perú 
Teléfono: 519900999445

Correo electrónico: adder413@gmail.com

Declaración de financiamiento y de conflicto de intereses: El presente artículo de revisión no requirió financiamiento de institución alguna.

Los autores declaran no tener conflictos de intereses.

\section{REFERENCIAS BIBLIOGRÁFICAS}

1. Duncan R, Razvi S, Mulhern S. Newly presenting psychogenic nonepileptic seizures: incidence, population characteristics, and early outcome from a prospective audit of a first seizure clinic. Epilepsy Behav. 2011;20(2):308-11. doi: 10.1016/j. yebeh.2010.10.022

2. Devinsky O, Gazzola D, LaFrance WC. Differentiating between nonepileptic and epileptic seizures. Nature reviews Neurology. 2011;7(4):210-20. doi:10.1038/ nrneurol.2011.24

3. Allendorfer JB, Nenert R, Hernando KA, DeWolfe JL, Pati S, Thomas AE, et al. FMRI response to acute psychological stress differentiates patients with psychogenic non-epileptic seizures from healthy controls - A biochemical and neuroimaging biomarker study. Neuroimage Clin. 2019;24:101967. doi:10.1016/j.nicl.2019.101967

4. Ortiz P. Introduccion a la Medicina Clínica: El Examen Neurológico Integral. Lima, Perú: Editorial Universidad Nacional Mayor de San Marcos; 1999. p. 69-75.

5. Tolchin B, Martino S, Hirsch LJ. Treatment of patients with psychogenic nonepileptic attackstreatment of patients with psychogenic nonepileptic attackstreatment of patients with psychogenic nonepileptic attacks. JAMA. 2019; 321(20): 19671968. doi: 10.1001/jama.2019.3520.

6. Robson C, Myers L, Pretorius C, Lian OS, Reuber M. Health related quality of life of people with nonepileptic seizures: The role of socio-demographic characteristics and stigma. Seizure. 2018;55:93-9. doi:10.1016/j.seizure.2018.01.001.

7. Bodde NM, Brooks JL, Baker GA, et al. Psychogenic non-epileptic seizures-definition, etiology, treatment and prognostic issues: a critical review. Seizure 2009;18:543-553.doi: 10.1016/j.seizure.2009.06.006.

8. Benbadis SR. Psychogenic nonepileptic "seizures" or "attacks"? It'snotjustsemantics: attacks. Neurology. 2010;75(1):84-6.doi: 10.1212/WNL.0b013e3181e6216f.

9. LaFrance WC, Baker GA, Duncan R, Goldstein LH, Reuber M. Minimum requirements for the diagnosis of psychogenic nonepileptic seizures: a staged approach: a report from the International League Against Epilepsy Nonepileptic Seizures Task Force. Epilepsia. doi: 10.1111/epi.12356.
10. Brigo F, Igwe SC, Ausserer H, Nardone R, Tezzon F, Bongiovanni LG, et al. Terminology of psychogenic nonepileptic seizures. Epilepsia. 2015;56(3):e21-5. doi: 10.1111/epi.12911

11. Hopp JL. Nonepileptic episodic events. Continuum (Minneap Minn). 2019; 25(2):492-507.doi: 10.1212/ CON.0000000000000711

12. Kerr MP, Mensah S, Besag F, de-Toffol B, Ettinger A, Kanemoto K, et al. International consensus clinical practice statements for the treatment of neuropsychiatric conditions associated with epilepsy. Epilepsia. 2011; 52(11):2133-8. doi:10.1111/j.15281167.2011.03276.x

13. Martin R, Burneo JG, Prasad A, Powell T, Faught E, Knowlton R, et al. Frequency of epilepsy in patients with psychogenic seizures monitored by video-EEG. Neurology. 2003;61(12):1791-2. doi:10.1212/01.wnl.0000098890.13946.f5

14. Benbadis SR, Allen Hauser W. An estimate of the prevalence of psychogenic non-epileptic seizures. Seizure. 2000; 9(4):280-1. doi:10.1053/ seiz.2000.0409

15. Asadi-Pooya A, Emami M. Juvenile and adultonset psychogenic non-epileptic seizures. Clin Neurol Neurosurg. 2013;115(9):1697-700. doi:10.1016/j.clineuro.2013.03.009

16. Asadi-Pooya AA. Psychogenic nonepileptic seizures are predominantly seen in women: potential neurobiological reasons. Neurol Sci. 2016;37(6):8515.doi:10.1007/s10072-016-2481-5.

17. Dworetzky B. The Impact of PNES is about more than counting events. Epilepsy Curr. 2016;16(5):314315. doi:10.5698/1535-7511-16.5.314

18. Karakis I, Cole AJ, Montouris GD, San Luciano M, Meador KJ, Piperidou C. Caregiver burden in epilepsy: determinants and impact. Epilepsy Res Treat. 2014; 2014:808421. doi:10.1155/2014/80842.

19. Marchetti RL, Kurcgant D, Gallucci-Neto J, Von-Bismark MA, Fiore LA. Epilepsy in patients with psychogenic non-epileptic seizures. Arq Neuropsiquiatr. 2010;68(2):168-73. doi:10.1590/ s0004-282x2010000200003

20. Silva W, Giagante B, Saizar R, D'Alessio L, Oddo $\mathrm{S}$, Consalvo D, et al. Clinical features and prognosis of nonepileptic seizures in a developing country. Epilepsia. 2001;42(3):398-401.doi: 10.1046/j.15281157.2001.45299.x

21. LaFrance WC, de-Marinis AJ, Webb AF, Machan JT, Rusch MD, Kanner AM. Comparing standard medical care for nonepileptic seizures in Chile and the United States. Epilepsy Behav. 2012;25(2):224-9. doi:10.1016/j.yebeh.2012.07.028

22. Alessi R, Valente KD. Psychogenic non-epileptic seizures at a tertiary care center in Brazil. Epilepsy Behav. 2013;26(1):91-5. doi: 10.1016/j. yebeh.2012.10.011 
Eventos no epilépticos psicógenos: El desafío constante de su diagnóstico y tratamiento.

23. Fiszman A, Alves-Leon SV, Nunes RG, D'Andrea I, Figueira I. Traumatic events and posttraumatic stress disorder in patients with psychogenic nonepileptic seizures: acritical review. EpilepsyBehav. 2004;5(6):818-25. doi:10.1016/j.yebeh.2004.09.002

24. D'Alessio L, Giagante B, Oddo S, Silva WW, Solis $\mathrm{P}$, Consalvo D, et al. Psychiatric disorders in patients with psychogenic non-epileptic seizures, with and without comorbid epilepsy. Seizure. 2006;15(5):3339. doi:10.1016/j.seizure.2006.04.003

25. Brown RJ, Reuber M. Towards an integrative theory of psychogenic non-epileptic seizures (PNES). Clin Psychol Rev. 2016;47:55-70. doi:10.1016/j. cpr.2016.06.003

26. Reuber M. The etiology of psychogenic nonepileptic seizures: toward a biopsychosocial model. Neurologic clinics. 2009;27(4):909-24. doi:10.1016/j. ncl.2009.06.004

27. Asadi-Pooya AA. Neurobiological origin of psychogenic nonepileptic seizures: A review of imaging studies. Epilepsy Behav. 2015;52(Pt A):2569. doi:10.1016/j.yebeh.2015.09.020

28. Arthuis M, Micoulaud-Franchi JA, Bartolomei F, McGonigal A, Guedj E. Resting cortical PET metabolic changes in psychogenic non-epileptic seizures (PNES). J Neurol Neurosurg Psychiatry. 2015;86(10):1106-12. doi:10.1136/jnnp-2014309390

29. Van-der-Kruijs SJ, Bodde NM, Vaessen MJ, Lazeron RH, Vonck K, Boon P, et al. Functional connectivity of dissociation in patients with psychogenic nonepileptic seizures. J Neurol Neurosurg Psychiatry. 2012;83(3):239-47. doi:10.1136/jnnp-2011-300776

30. Ding J, An D, Liao W, Wu G, Xu Q, Zhou D, et al. Abnormal functional connectivity density in psychogenic non-epileptic seizures. Epilepsy Res. 2014;108(7):1184-94. doi:10.1016/j. eplepsyres.2014.05.006

31. Reuber M, Brown RJ. Understanding psychogenic nonepileptic seizures-Phenomenology, semiology and the Integrative Cognitive Model. Seizure. 2017; 44: 199-205. doi:10.1016/j.seizure.2016.10.029

32. Avbersek A, Sisodiya S. Does the primary literature provide support for clinical signs used to distinguish psychogenic nonepileptic seizures from epileptic seizures? Journal of neurology, neurosurgery, and psychiatry. 2010;81(7):719-25. doi:10.1136/ jnnp.2009.197996

33. Erba G, Giussani G, Juersivich A, Magaudda A, Chiesa V, Lagana A, et al. The semiology of psychogenic nonepileptic seizures revisited: Can video alone predict the diagnosis? Preliminary data from a prospective feasibility study. Epilepsia. 2016;57(5):777-85. doi: 10.1111/epi.13351

34. Hubsch C, Baumann C, Hingray C, Gospodaru N, Vignal JP, Vespignani H, et al. Clinical classification of psychogenic non-epileptic seizures based on video-EEG analysis and automatic clustering. J Neurol Neurosurg Psychiatry. 2011;82(9): 955-60. doi:10.1136/jnnp.2010.235424

35. Seneviratne U, Reutens D, D'Souza W. Stereotypy of psychogenic nonepileptic seizures: insights from video-EEG monitoring. Epilepsia. 2010;51(7):115968. doi:10.1111/j.1528-1167.2010.02560.x

36. van-der-Kruijs SJ, Vonck KE, Langereis GR, Feijs LM, Bodde NM, Lazeron RH, et al. Autonomic nervous system functioning associated with psychogenic nonepileptic seizures: Analysis of heart rate variability. Epilepsy Behav. 2016;54:14-9. doi:10.1016/j.yebeh.2015.10.014

37. Asadi-Pooya AA, Bahrami Z. Auras in psychogenic nonepileptic seizures. Seizure. 2019;69:215-7. doi:10.1016/j.seizure.2019.05.012

38. Plug L, Sharrack B, Reuber M. Seizure metaphors differ in patients' accounts of epileptic and psychogenic nonepileptic seizures. Epilepsia. 2009;50(5):9941000. doi:10.1111/j.1528-1167.2008.01798.x

39. Robson C, Drew P, Walker T, Reuber M. Catastrophising and normalising in patient's accounts of their seizure experiences.Seizure. 2012;21(10): 795-801. doi:10.1016/j.seizure.2012.09.007

40. Krumholz A, Hopp J. Psychogenic (nonepileptic) seizures. Seminars in neurology. 2006;26(3):341-50. doi: $10.1055 / \mathrm{s}-2006-945520$

41. Syed TU, LaFrance WC, Kahriman ES, Hasan SN, Rajasekaran V, Gulati D, et al. Can semiology predict psychogenic nonepileptic seizures? A prospective study. Annals of neurology. 2011;69(6):997-1004. doi:10.1002/ana.22345

42. Shih JJ, Fountain NB, Herman ST, Bagic A, Lado F, Arnold S, et al. Indications and methodology for video-electroencephalographic studies in the epilepsy monitoring unit. Epilepsia. 2018;59(1):2736. doi:10.1111/epi.13938

43. Mayor L, Burneo J, Ochoa J. Generadores del electroencefalograma. En: Mayor L, Burneo J, Ochoa J. Manual de Electroencefalografía. 1ra.ed. Colombia:Universidad de los Andes; 2013.p.3-7.

44. Meppelink AM, Parees I, Beudel M, Little S, Yogarajah M, Sisodiya S, et al. Spectral power changes prior to psychogenic non-epileptic seizures: a pilot study. J Neurol Neurosurg Psychiatry. 2017;88(2):1902. doi:10.1136/jnnp-2016-314080

45. Drane DL, LaRoche SM, Ganesh GA, Teagarden D, Loring DW. A standardized diagnostic approach and ongoing feedback improves outcome in psychogenic nonepileptic seizures. 2016;54:34-9. doi:10.1016/j.yebeh.2015.10.026

46. Gale SD, Hill SW. Concurrent administration of the MMPI-2 and PAI in a sample of patients with epileptic or non-epileptic seizures: implications for an inpatient epilepsy monitoring unit. Epilepsy 
\& behavior. 2012;25(2):181-4.doi: 10.1016/j.yebeh. 2012.07.012

47. McSweeney M, Reuber M, Levita L. Neuroimaging studies in patients with psychogenic non-epileptic seizures: A systematic meta-review. NeuroImage Clinical. 2017;16:210-21.doi: 10.1016/j.nicl.2017. 07.025

48. Nass RD, Sassen R, Elger CE, Surges R. The role of postictal laboratory blood analyses in the diagnosis and prognosis of seizures. Seizure. 2017; 47: 51-65. doi:10.1016/j.seizure.2017.02.013

49. Matz O, Heckelmann J, Zechbauer S, Litmathe J, Brokmann JC, Willmes K, et al. Early postictal serum lactate concentrations are superior to serum creatine kinase concentrations in distinguishing generalized tonic-clonic seizures from syncopes. Intern Emerg Med. 2018;13(5):749-55. doi: 10.1007/s11739-0171745-2.

50. Beniczky S, Conradsen I, Pressler R, Wolf P. Quantitative analysis of surface electromyography: Biomarkers for convulsive seizures. Clin Neurophysiol. 127(8):2900-07,2016. doi:10.1016/j. clinph.2016.04.017

51. Beniczky S, Conradsen I, Moldavo M, Jennum P, Fabricius M, Benedek K, et al. Quantitative analysis of surface electromyography during epileptic and nonepileptic convulsive seizures. Epilepsia. 2014; 55(7):1128-1134. doi: 10.1111/epi.12669
52. Beniczky S, Conradsen I, Moldovan M, Jennum P, Fabricius M, Benedek $\mathrm{K}$, et al. Automated differentiation between epileptic and nonepileptic convulsive seizures. Ann Neurol. 2015; 77: 348-351. doi:10.1002/ana.24338

53. Gasparini S, Beghi E, Ferlazzo E, Beghi M, Belcastro V, Biermann KP, et al. Management of psychogenic non-epileptic seizures: a multidisciplinary approach. Eur J Neurol. 2019;26(2):205-e15. doi: 10.1111/ ene. 13818

54. Asadi-Pooya AA. Psychogenic nonepileptic seizures: a concise review. Neurol Sci. 2017;38(6):935-40. doi:10.1007/s10072-017-2887-8

55. Reuber M, Pukrop R, Bauer J, Helmstaedter C, Tessendorf N, Elger CE. Outcome in psychogenic nonepileptic seizures: 1 to 10-year follow-up in 164 patients. Ann Neurol. 2003;53(3):305-11. doi:10.1002/ana.3000

56. Durrant J, Rickards H, Cavanna AE. Prognosis and outcome predictors in psychogenic nonepileptic seizures. Epilepsy research and treatment. 2011; 2011: 274736. doi:10.1155/2011/274736

Recibido: 07/01/2020

Aceptado: 16/08/2020 